\title{
LA BIOMASA DE LOS SISTEMAS PRODUCTIVOS DE MAÍZ NATIVO (Zea mays) COMO ALTERNATIVA A LA CAPTURA DE CARBONO
}

\author{
Beatriz MARCOS SOLORIO ${ }^{1}$, Ángel Roberto MARTÍNEZ CAMPOS ${ }^{1}$, \\ Guadalupe Alfonso LÓPEZ URQUÍDEZ ${ }^{2}$, Carlos Alfonso LÓPEZ ORONA ${ }^{2 *}$ y \\ Tizbe Teresa ARTEAGA REYES ${ }^{1}$
}

${ }^{1}$ Instituto de Ciencias Agropecuarias y Rurales, Universidad Autónoma del Estado de México. El Cerrillo Piedras Blancas, Toluca, Estado de México, México, C.P. 50090

${ }^{2}$ Facultad de Agronomía, Universidad Autónoma de Sinaloa. Carretera Culiacán-Eldorado km. 17.5, Culiacán de Rosales, Sinaloa, México, C.P. 80398

*Autor para correspondencia: clopezorona@uas.edu.mx

(Recibido junio 2015; aceptado noviembre 2015)

Palabras clave: cambio climático, fijación de carbono, equivalentes de carbono, sistemas agrícolas, ambientes contrastantes

\section{RESUMEN}

Con la finalidad de obtener información cuantitativa sobre el potencial de fijación de carbono en cultivos de maíz, se evaluaron cuatro variedades nativas del mismo (negro, amarillo, blanco y cacahuacintle), bajo tres ambientes contrastantes (montaña, valle y planicie). En el ambiente de montaña (Jiquipilco, Estado de México) la temperatura y precipitación media durante el ciclo del cultivo fue de $16^{\circ} \mathrm{C}$ y $818.5 \mathrm{~mm}$ respectivamente, y en el valle (Temoaya, Estado de México) fue de $16^{\circ} \mathrm{C}$ y $768.8 \mathrm{~mm}$ respectivamente. Las parcelas fueron sembradas el 8 de mayo de 2013. Los cultivos de planicie (Culiacán, Sinaloa) se sembraron el día 19 de diciembre del 2013 y la temperatura y precipitación media en éstos fue de $23^{\circ} \mathrm{C}$ y $44.1 \mathrm{~mm}$ respectivamente. El análisis estadístico indicó que la concentración de carbono entre variedades es estadísticamente diferente $(\mathrm{P}<0.05)$, la variedad amarilla obtuvo el valor más alto $\left(34.3 \mathrm{tCO}_{2} \mathrm{eq} / \mathrm{ha}\right)$, mientras que las variedades negro y cacahuacintle registraron la capacidad más baja de fijación de carbono, con un promedio de $24.5 \mathrm{tCO}_{2}$ eq/ha para cada una. Los ambientes de montaña y de valle resultaron con los mayores valores de fijación de carbono (41.6 $\left.\mathrm{tCO}_{2} \mathrm{eq} / \mathrm{ha}\right)$, comparados con los de planicie (11.4 $\mathrm{tCO}_{2} \mathrm{eq} / \mathrm{ha}$ ) y fueron estadísticamente diferentes. Se concluye que los sistemas productivos de maíz con variedades nativas pueden considerarse como una alternativa para la fijación del carbono.

Key words: climate change, carbon sequestration, carbon equivalents, farming systems, contrasting environments

\begin{abstract}
In order to obtain quantitative information on the carbon sequestration potential of corn, four native maize varieties (black, yellow, white and cacahuacintle) under three contrasting environments (mountain, valley and plain) were evaluated. In the mountain environment (Jiquipilco, State of Mexico) the temperature and rainfall during the crop cycle was $16^{\circ} \mathrm{C}$ and $818.5 \mathrm{~mm}$, respectively and in the valley (Temoaya, State of
\end{abstract}


Mexico) was $16^{\circ} \mathrm{C}$ and $768.8 \mathrm{~mm}$, respectively. Plots were seeded on may 8,2013 . The plain crops (Culiacan, Sinaloa) were seeded on december 19, 2013, and the average temperature and precipitation was $23^{\circ} \mathrm{C}$ and $44.1 \mathrm{~mm}$ respectively. Statistical analysis indicated that the concentration of carbon between varieties is statistically different $(\mathrm{P}<0.05)$. The yellow variety had the highest value $\left(34.3 \mathrm{tCO}_{2} \mathrm{eq} / \mathrm{ha}\right)$, while the black and cacahuacintle varieties had the lowest binding capacity, with an average of 24.5 $\mathrm{tCO}_{2} \mathrm{eq} / \mathrm{ha}$ for each. The mountain and valley environments presented the highest values of carbon fixing (41.6 tCO $\left.\mathrm{tC}_{2} \mathrm{eq} / \mathrm{ha}\right)$ compared to those of plain $\left(11.4 \mathrm{tCO}_{2} \mathrm{eq} / \mathrm{ha}\right)$, and they were also statistically different. It is concluded that production systems with native corn varieties can be considered as an alternative to carbon fixation.

\section{INTRODUCCIÓN}

El cambio climático es un problema ambiental que se ha agravado considerablemente en las últimas décadas debido a un aumento de los gases de efecto invernadero emitidos hacia la atmósfera como resultado de la actividad humana. Dentro de estos gases el que mayor aumento de concentración ha tenido es el dióxido de carbono $\left(\mathrm{CO}_{2}\right)$. Algunas alternativas para disminuir su concentración son a través del secuestro de carbono y de su fijación en el suelo o de su almacenado en forma de biomasa, la cual incluye todo tipo de vegetación y organismos vivos de todos los ecosistemas (Espinoza et al. 2012).

Los sistemas agrícolas, además de ser una fuente de alimento, han demostrado tener capacidad de captura de $\mathrm{CO}_{2}$ (Robert 2002), lo cual depende de la productividad de los mismos sistemas y de las condiciones ambientales bajo las que se cultiva. En México los cultivos de maíz nativo poseen una gran diversidad genética. El $80 \%$ de la superficie cultivada en el país corresponde a maíz nativo y el $20 \%$ a maíces mejorados (Aragón et al. 2006). Estos cultivos se encuentran principalmente en condiciones de temporal y los agricultores generalmente disponen de más de una variedad nativa adaptada a su ambiente (Aceves et al. 2002).

En concreto, el cultivo de maíz por medio de la fotosíntesis asimila el $\mathrm{CO}_{2}$ atmosférico, lo transforma en carbohidratos y lo fija en sus estructuras para aumentar su biomasa (Espinoza et al. 2012). El carbono orgánico del suelo (COS) está en función de la tasa de descomposición de los residuos de las cosechas, como raíces y otros materiales orgánicos que retornan al suelo en la misma cantidad y composición (Follett 2001). Parte de este COS regresa a la atmósfera a causa de la respiración celular y a la descomposición de la materia orgánica del suelo. Es así como los cultivos permiten incorporar el carbono atmosférico al ciclo biológico (Casanova et al. 2011).

El objetivo del presente trabajo fue determinar el potencial de fijación de $\mathrm{CO}_{2}$ en la biomasa de los sistemas productivos de cuatro variedades de maíz nativo del Estado de México en tres ambientes diferentes (montaña, valle y planicie).

\section{MATERIALES Y MÉTODOS}

\section{Establecimiento de los cultivos}

Cuatro variedades de maíz fueron evaluadas en cuanto a su capacidad de fijación de carbono: maíz blanco, amarillo, negro y cacahuacintle, las que tienen aproximadamente 70 años de cultivarse en Temoaya y Jiquipilco, Estado de México (Fig. 1). Los cultivos se sembraron en tres ambientes contrastantes: montaña, valle y planicie, bajo diferentes condiciones de manejo. La parcela de montaña se ubicó en el municipio de Jiquipilco, que se localiza en la parte central del Estado de México a una altitud de $2750 \mathrm{msnm}$, el clima fue templado subhúmedo con lluvias en verano, con temperatura y precipitación media durante el ciclo de cultivo de $16^{\circ} \mathrm{C}$ y $818.5 \mathrm{~mm}$, respectivamente (CONAGUA 2015). Los cultivos de valle se establecieron en el municipio de Temoaya, el cual se localiza en la parte centro norte del Estado de México a una altitud de $2680 \mathrm{msnm}$, el clima es templado

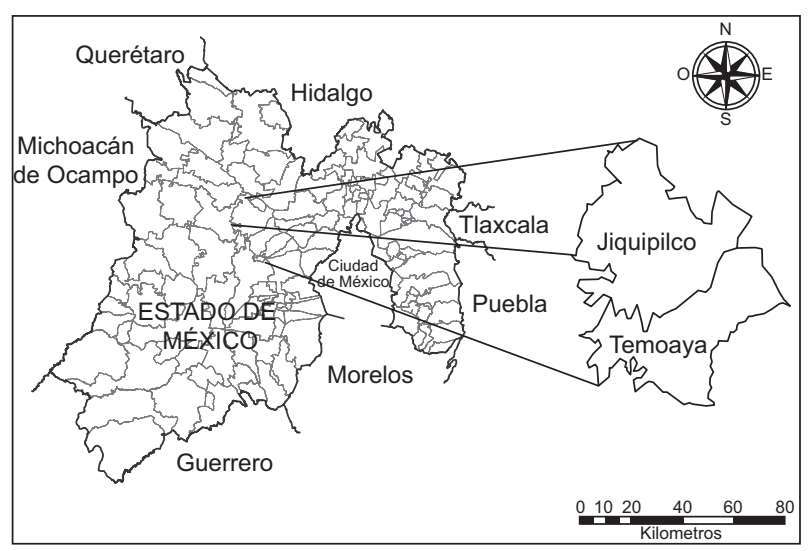

Fig. 1. Localización de los municipios de Jiquipilco y Temoaya 
subhúmedo con lluvias en verano, con temperatura y precipitación media durante el cultivo de $16{ }^{\circ} \mathrm{C}$ y $768.8 \mathrm{~mm}$, respectivamente (CONAGUA 2015). Los cultivos de montaña y valle fueron establecidos el 8 de mayo de 2013 en una superficie de 1 ha para cada ambiente. Se mantuvieron como cultivos de temporal con un riego de auxilio. La distancia entre surcos fue de $80 \mathrm{~cm}$, distancia entre plantas de $20 \mathrm{~cm}$ y se aplicó una fertilización química en la presiembra de $136 \mathrm{~kg}$ de nitrógeno (300 kg urea). Para el caso de los cultivos de valle, adicionalmente se aplicaron 150 t de estiércol de bovino. El ambiente de planicie se estableció en el campo experimental de la Facultad de Agronomía de la Universidad Autónoma de Sinaloa, localizado en el municipio de Culiacán (Fig. 2) a una altitud de 19 msnm, el clima predominante fue seco-cálido, con temperatura y precipitación media durante el ciclo del cultivo de $23{ }^{\circ} \mathrm{C}$ y $44.1 \mathrm{~mm}$, respectivamente (CONAGUA 2015). Este cultivo se sembró el 19 de diciembre de 2013 en una superficie de 1 ha. Se mantuvo la misma densidad de plantación descrita anteriormente. Se realizó una fertilización química orgánica en la presiembra con $80 \mathrm{~kg}$ de urea y $50 \mathrm{~kg}$ de fósforo, utilizando como fuente de los nutrientes urea y fosfáto monoamónico. Asimismo, también se empleó una tonelada de composta. Se aplicó una segunda fertilización a los 40 días posteriores a la siembra de $200 \mathrm{~kg}$ de nitrógeno. Los riegos se realizaron a través de un sistema de multicompuertas, el primer riego de auxilio se aplicó a los 45 días después de la siembra, el segundo a los 65, el tercero a los 85 y el cuarto a los 105 días después de la siembra.

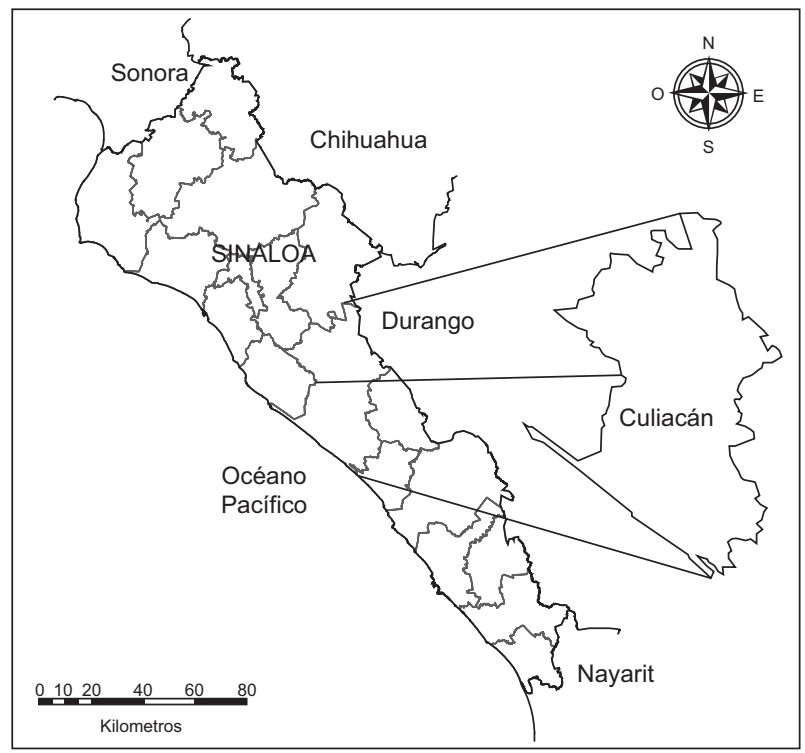

Fig. 2. Localización del municipio de Culiacán
La siembra se hizo de manera manual en las tres zonas, se mantuvo la densidad de 62000 plantas por ha, con un índice de pérdida del $5 \%$, el cual fue obtenido mediante una prueba de germinación. En los cultivos sembrados en el ambiente de planicie se aplicó una dosis de $150 \mathrm{~mL} / \mathrm{ha}$ del producto Denim 19 CE de Syngenta (benzoato de emamectina $2.12 \%$ ) para el control del gusano cogollero (Spodoptera frugiperda). La fumigación fue dirigida al cogollo de la planta con una bomba de aspersión manual. En los cultivos de montaña y valle no se presentó la plaga de este gusano cogollero.

\section{Muestreo y evaluaciones morfométricas}

Se hicieron muestreos aleatorios de 36 plantas por cada ambiente y variedades cuando alcanzaron su madurez fisiológica (140 días después de la siembra), para determinar la altura de la planta, la longitud del tallo, de la raíz, de la espiga y de la mazorca, así como el peso de las hojas. La altura se midió desde la base del tallo hasta la punta de la última hoja expuesta de la planta, con una regla de medición. La longitud del tallo se midió desde la base del tallo hasta donde inicia la espiga, finalmente la longitud de la espiga se consideró como la parte restante. La raíz fue extraída completamente del suelo y se limpió con agua para medir su longitud con una regla de medición. La mazorca fue cortada del tallo y después se le desprendieron las hojas protectoras para medir su longitud y masa. Se colocó el total de las hojas por planta en una bolsa de papel y se secaron en una estufa a $70{ }^{\circ} \mathrm{C}$ por $24 \mathrm{~h}$ para determinar la pérdida de peso.

\section{Composición química del maíz}

La estimación del carbono fijado se realizó a partir la composición química proximal de cada estructura. Esta composición química fue determinada mediante análisis bromatológicos (AOAC 2006), que incluyeron contenidos de humedad, proteína, fibra y ceniza del material vegetal colectado en campo. Para el caso del grano, adicionalmente se determinó almidón por la técnica de textura por disección (Pérez et al. 2007).

\section{Equivalentes de $\mathrm{CO}_{2}$ fijados en la biomasa}

Para determinar el porcentaje correspondiente a carbono en cada compuesto, se utilizaron las siguientes expresiones:

$\% \mathrm{C}=\mathrm{PMC} / \mathrm{PMDC} * 100$

$\mathrm{CT}=\left((\% \mathrm{C})^{*}(\mathrm{gCM}) / 100\right)^{*}$

$\mathrm{EqCO}_{2}=(\mathrm{CT} * 44) / 12$ 
dónde: $\% \mathrm{C}=$ porcentaje de carbono correspondiente a cada compuesto, (12 almidón, 24 proteína y 72 para fibra) $\mathrm{PMC}=$ peso molecular del carbono contenido en cada compuesto determinado a partir de la fórmula mínima, $\mathrm{PMDC}=$ Peso molecular de la fórmula mínima de cada compuesto (almidón $30 \mathrm{~g} / \mathrm{mol}$, proteína $75 \mathrm{~g} / \mathrm{mol}$, fibra $162 \mathrm{~g} / \mathrm{mol}), \mathrm{CT}=$ carbono total, $\mathrm{gCM}=$ gramos del compuesto (almidón, proteína, fibra) en la muestra, PS = peso seco de la muestra, $\mathrm{EqCO}_{2}=$ equivalentes de $\mathrm{CO}_{2}$. La determinación del carbono fijado se presenta en toneladas de equivalentes de $\mathrm{CO}_{2}$ por hectárea ( $\left.\mathrm{tCO}_{2} \mathrm{eq} / \mathrm{ha}\right)$.

\section{Análisis estadístico}

El diseño experimental empleado para el análisis de los resultados fue bloques completamente al azar, donde cada ambiente fue considerado un tratamiento y cada variedad un bloque. Se realizaron pruebas de Kolmogorov-Smirnov para identificar desviaciones respecto a la distribución normal. Una vez que se comprobó la normalidad en los datos, se realizó un análisis de varianza y posteriormente una prueba de comparación de medias de Tukey $(\mathrm{P} \leq 0.05)$. Se utilizó el programa estadístico SAS (Statistical Analysis System 1998).

\section{RESULTADOS Y DISCUSIÓN}

\section{Evaluaciones morfométricas por ambiente}

Los valores medios de las longitudes del tallo, raíz, mazorca y peso de las hojas cultivadas en el valle y en la montaña fueron $1.4,1.8,1.4,2.0$ veces más que los alcanzados en las plantas de planicie y fueron estadísticamente diferentes ( $\mathrm{P} \leq 0.05$; Cuadro I). Estos resultados son consistentes con los mayores rendimientos de grano obtenidos en los cultivos de montaña y valle 8.40 y $9.00 \mathrm{t} / \mathrm{ha}$, respectivamente, al compararlos con los de planicie (1.69 t/ha),

\begin{tabular}{lccccc} 
CUADRO I. & MEDIDAS MORFOMÉTRICAS EN PLANTAS \\
DE MAÍZ EN TRES AMBIENTES DIFEREN- \\
TES \\
$\begin{array}{lccccc}\text { Tipo de } \\
\text { ambiente }\end{array}$ & $\begin{array}{c}\text { LT } \\
(\mathrm{cm})\end{array}$ & $\begin{array}{c}\text { LR } \\
(\mathrm{cm})\end{array}$ & $\begin{array}{c}\text { LM } \\
(\mathrm{cm})\end{array}$ & $\begin{array}{c}\text { PH } \\
(\mathrm{g})\end{array}$ & $\begin{array}{c}\text { PG } \\
(\mathrm{g})\end{array}$ \\
\hline Planicie & $218.0 \mathrm{a}$ & $16.7 \mathrm{a}$ & $10.8 \mathrm{a}$ & $30.8 \mathrm{a}$ & $28.1 \mathrm{a}$ \\
Valle & $308.0 \mathrm{~b}$ & $30.6 \mathrm{c}$ & $15.2 \mathrm{~b}$ & $61.7 \mathrm{~b}$ & $149.9 \mathrm{~b}$ \\
Montaña & $295.7 \mathrm{~b}$ & $25.7 \mathrm{~b}$ & $16.4 \mathrm{~b}$ & $61.5 \mathrm{~b}$ & $139.9 \mathrm{~b}$ \\
\hline
\end{tabular}

Valores con la misma letra por columna son estadísticamente iguales, significancia de 0.05 . $\mathrm{LT}=$ Longitud de tallo, $\mathrm{LR}=$ Longitud de raíz, $\mathrm{LM}=$ Longitud de mazorca, $\mathrm{PH}=$ Peso de hojas, $\mathrm{PG}=$ Peso de grano. ya que el rendimiento está asociado a la longitud de la raíz, el tallo y las hojas (Paliwal 2001). Lo anterior coincide con lo reportado por Pecina et al. (2011), quienes encontraron que a mayor altitud y menor temperatura se incrementa el rendimiento de grano del maíz. Observaron que los cultivos de valles altos superaron a los de transición y éstos a los de trópico seco, probablemente por problemas de adaptación, lo que implica una menor capacidad para las plantas de expresar su máximo potencial de rendimiento.

\section{Evaluaciones morfométricas por variedad}

Las longitudes medias de tallo, raíz, mazorca, espiga y peso de las hojas fueron de $270 \mathrm{~cm}, 23.7 \mathrm{~cm}$, $14.1 \mathrm{~cm}, 24.2 \mathrm{~cm}$ y $50.3 \mathrm{~g}$, respectivamente y no presentaron diferencias estadísticas entre variedades $(\mathrm{P} \leq 0.05)$. Estos valores son superiores a los reportados por Espinosa et al. (2013) en plantas de variedades criollas amarillas en valles altos de $2.2 \mathrm{~m}$ con una longitud de mazorca de $13.7 \mathrm{~cm}$.

Además, estas variedades nativas superan en longitud a los maíces híbridos de valles altos (2200-2274 $\mathrm{msnm}$ ), cuyo rango es de 190 a $262 \mathrm{~cm}$ (Arellano et al. 2010, Tadeo et al. 2012).

Elizondo y Boschini (2002) reconocen que el cultivar maíz criollo permite obtener una mayor producción de forraje verde y seco (> $30 \%$ ) por ha, ya que la altura promedio de la planta del maíz criollo puede superar a las plantas de variedades híbridas en $1.7 \mathrm{~m}$.

\section{Análisis de composición química por variedad}

El mayor porcentaje de proteína se encontró en el grano de la variedad amarilla con $7.71 \%$ y el menor porcentaje fue de $2.60 \%$ en la hoja de la mazorca de la misma variedad (Cuadro II). Sin embargo, el porcentaje de proteína obtenido en el grano es menor a lo reportado por Vera et al. (2012) quienes encontraron porcentajes de proteína de $11.9 \%$ para grano en maíz amarillo.

En cultivos de maíz criollo forrajero, Amador et al. (2000) registraron porcentajes de proteína en tallo de $7.3 \%$, estos valores superan a los obtenidos en el presente estudio, ya que el tallo de la variedad negra obtuvo $6.24 \%$ de proteína y fue superior al resto de las variedades con diferencias significativas $(\mathrm{P} \leq 0.05)$. El valor medio del porcentaje de proteína en hoja, raíz y hoja de mazorca fue $4.3 \%$ $\mathrm{y}$ no se encontraron diferencias entre variedades $(\mathrm{P} \leq 0.05)$.

En contraste, los porcentajes de fibra en tallo fueron superiores a los reportados por Amador et al. (2000), quien obtuvo $67.03 \%$, en nuestro estudio la 
CUADRO II. PORCENTAJE DE PROTEÍNA Y FIBRA DE CADA ESTRUCTURA DE LA PLANTA EN DIFERENTES VARIEDADES DE MAÍZ NATIVO

\begin{tabular}{|c|c|c|c|c|c|c|c|c|c|c|}
\hline \multirow{3}{*}{ Variedad } & \multicolumn{10}{|c|}{ Estructuras de la planta } \\
\hline & \multicolumn{2}{|c|}{ Tallo } & \multicolumn{2}{|c|}{ Hoja } & \multicolumn{2}{|c|}{ Raíz } & \multicolumn{2}{|c|}{ Hoja de mazorca } & \multicolumn{2}{|c|}{ Grano } \\
\hline & Proteína & Fibra & Proteína & Fibra & Proteína & Fibra & Proteína & Fibra & Proteína & Fibra \\
\hline Negro & $6.2 \mathrm{~b}$ & $83.2 \mathrm{~b}$ & $6.3 \mathrm{a}$ & $80.2 \mathrm{~b}$ & $3.9 \mathrm{a}$ & $72.6 \mathrm{ab}$ & $2.7 \mathrm{a}$ & $80.7 \mathrm{a}$ & $6.9 \mathrm{ab}$ & $9.4 \mathrm{a}$ \\
\hline Cacahuacintle & $5.7 \mathrm{ab}$ & $81.9 \mathrm{~b}$ & $6.8 \mathrm{a}$ & $80.1 \mathrm{~b}$ & $3.6 \mathrm{a}$ & $71.9 \mathrm{ab}$ & $3.0 \mathrm{a}$ & $78.7 \mathrm{a}$ & $6.7 \mathrm{a}$ & $8.3 \mathrm{a}$ \\
\hline Blanco & $4.0 \mathrm{a}$ & $84.2 \mathrm{~b}$ & $5.5 \mathrm{a}$ & $74.3 \mathrm{a}$ & $2.8 \mathrm{a}$ & $69.0 \mathrm{a}$ & $2.8 \mathrm{a}$ & $78.2 \mathrm{a}$ & $7.2 \mathrm{ab}$ & $13.3 \mathrm{a}$ \\
\hline Amarillo & $5.91 \mathrm{ab}$ & $75.7 \mathrm{a}$ & $6.9 \mathrm{a}$ & $78.6 \mathrm{ab}$ & $4.1 \mathrm{a}$ & $75.2 \mathrm{~b}$ & $2.6 \mathrm{a}$ & $80.7 \mathrm{a}$ & $7.7 \mathrm{~b}$ & $10.3 \mathrm{a}$ \\
\hline
\end{tabular}

Valores con la misma letra por columna son estadísticamente iguales, significancia de 0.05

variedad blanca registró $84.28 \%$. De igual forma el porcentaje de fibra en grano blanco $(13.39 \%)$ superó los porcentajes de 7.1 y $13.1 \%$ reportados por Méndez et al. (2005) en variedades híbridas y 10.10 $\%$ en maíz criollo (Cuadro II). Estas diferencias en composición química se deben a la gran diversidad genética del maíz, que se refleja en sus diferentes propiedades y utilización.

\section{Análisis de composición química por ambiente}

Los porcentajes de proteína del tallo, hoja, raíz, hoja de la mazorca y grano de las plantas cultivadas en el valle fueron $1.2,2.3,3.5,2.4$ y 1.2 veces mayores y estadísticamente diferentes $(\mathrm{P} \leq 0.05)$ a las obtenidas en los cultivos de planicie. Sin embargo, los porcentajes de fibra disminuyeron (Cuadro III). Estos resultados son consistentes con lo señalado por Zepeda et al. (2009) quienes hacen referencia a un estudio realizado por Zhang et al. (1993), donde en maíces cultivados con dosis altas de nitrógeno se modificó la composición del grano. Dichos autores evaluaron el efecto de 0,90 y $180 \mathrm{~kg} /$ ha de nitrógeno por tres años, en cuatro localidades y encontraron que los porcentajes de fibra y celulosa disminuyeron al aumentar las dosis de nitrógeno.
Los mayores porcentajes de fibra se presentaron en los cultivos de montaña, con excepción de la hoja de mazorca y grano donde los cultivos de valle y planicie obtuvieron los mayores porcentajes ( $81.65 \% \mathrm{y}$ $18.29 \%$ respectivamente) (Cuadro III).

\section{Fijación de carbono por variedad}

El análisis de varianza mostró que existen diferencias significativas entre la fijación de equivalentes de $\mathrm{CO}_{2}$ en las diferentes variedades $(\mathrm{P} \leq 0.05)$. Las que obtuvieron el porcentaje menor fueron las variedades negra y cacahuacintle con 24.77 y $24.26 \mathrm{tCO}_{2} \mathrm{eq} / \mathrm{ha}$, respectivamente comparadas con la variedad amarilla que obtuvo 1.4 veces más $\left(34.37 \mathrm{tCO}_{2} \mathrm{eq} / \mathrm{ha}\right.$; Cuadro IV).

\section{Fijación de carbono por ambiente}

En los cultivos de montaña se encontró la mayor cantidad de equivalentes de $\mathrm{CO}_{2}$ fijado con más de $41 \mathrm{tCO}_{2} \mathrm{eq} / \mathrm{ha}, 1.4$ veces más que los cultivos de valle y 3.6 veces más que los de planicie. El mayor contenido de carbono fijado fue en el tallo, en cultivos de montaña esta estructura fijó 4.9 veces más de equivalentes de carbono que los tallos de los cultivos de planicie (3.80 $\mathrm{tCO}_{2}$ eq/ha; Cuadro V).

CUADRO III. PORCENTAJE DE PROTEÍNA Y FIBRA DE CADA ESTRUCTURA EN PLANTAS DE MAÍZ NATIVO PRODUCIDO EN DIFERENTES AMBIENTES

\begin{tabular}{|c|c|c|c|c|c|c|c|c|c|c|}
\hline \multirow{3}{*}{ Ambiente } & \multicolumn{10}{|c|}{ Estructuras de la planta } \\
\hline & \multicolumn{2}{|c|}{ Tallo } & \multicolumn{2}{|c|}{ Hoja } & \multicolumn{2}{|c|}{ Raíz } & \multicolumn{2}{|c|}{ Hoja de mazorca } & \multicolumn{2}{|c|}{ Grano } \\
\hline & Proteína & Fibra & Proteína & Fibra & Proteína & Fibra & Proteína & Fibra & Proteína & Fibra \\
\hline Planicie & $5.5 \mathrm{a}$ & $81.3 \mathrm{~b}$ & $3.9 \mathrm{a}$ & $75.8 \mathrm{a}$ & $1.5 \mathrm{a}$ & $70.3 \mathrm{a}$ & $1.5 \mathrm{a}$ & $78.4 \mathrm{a}$ & $6.6 \mathrm{a}$ & $18.2 b$ \\
\hline Valle & $7.0 \mathrm{~b}$ & $75.9 \mathrm{a}$ & $8.9 \mathrm{c}$ & $76.8 \mathrm{a}$ & $5.4 \mathrm{c}$ & $72.2 \mathrm{ab}$ & $3.7 \mathrm{~b}$ & $81.6 b$ & $7.7 \mathrm{~b}$ & $4.9 \mathrm{a}$ \\
\hline Montaña & $4.6 \mathrm{a}$ & $84.5 \mathrm{~b}$ & $7.2 \mathrm{~b}$ & $82.9 \mathrm{~b}$ & $4.6 \mathrm{~b}$ & $74.8 \mathrm{~b}$ & $3.3 \mathrm{~b}$ & $79.6 \mathrm{ab}$ & $7.1 \mathrm{ab}$ & $5.7 \mathrm{a}$ \\
\hline
\end{tabular}

Valores con la misma letra por columna son estadísticamente iguales, significancia de 0.05 
CUADRO IV. EQUIVALENTES DE $\mathrm{CO}_{2}$ FIJADOS POR CADA ESTRUCTURA DE LA PLANTA Y VARIEDAD DE MAÍZ NATIVO $\left(\mathrm{tCO}_{2} \mathrm{eq} / \mathrm{ha}\right)$

\begin{tabular}{lrrrrrc}
\hline Variedad de maíz & Tallo & Hoja & Raíz & Hoja de la mazorca & Grano & Total \\
\hline Cacahuacintle & $8.1 \mathrm{a}$ & $4.4 \mathrm{a}$ & $1.8 \mathrm{a}$ & $1.8 \mathrm{a}$ & $8.4 \mathrm{a}$ & $24.7 \mathrm{a}$ \\
Negro & $8.3 \mathrm{a}$ & $3.9 \mathrm{a}$ & $2.0 \mathrm{ab}$ & $1.7 \mathrm{a}$ & $8.1 \mathrm{a}$ & $24.2 \mathrm{a}$ \\
Blanco & $11.0 \mathrm{a}$ & $3.8 \mathrm{a}$ & $2.3 \mathrm{ab}$ & $2.1 \mathrm{a}$ & $7.0 \mathrm{a}$ & $26.4 \mathrm{~b}$ \\
Amarillo & $13.9 \mathrm{a}$ & $4.2 \mathrm{a}$ & $3.0 \mathrm{~b}$ & $2.0 \mathrm{a}$ & $11.0 \mathrm{a}$ & $34.3 \mathrm{c}$ \\
\hline
\end{tabular}

Valores con la misma letra por columna son estadísticamente iguales, significancia de 0.05

\begin{tabular}{lrccccc} 
CUADRO V. EQUIVALENTES DE $\mathrm{CO}_{2}$ & FIJADOS POR \\
& CADA ESTRUCTURA DE PLANTAS DE MAÍZ \\
& $\begin{array}{l}\text { NATIVO PRODUCIDA EN DIFERENTES AM- } \\
\text { BIENTES (tCO }\end{array}$ eq/ha) \\
\hline Ambiente & Tallo & Hoja & Raíz & $\begin{array}{c}\text { Hoja de la } \\
\text { mazorca }\end{array}$ \\
\hline Planicie & $3.7 \mathrm{a}$ & $2.3 \mathrm{a}$ & $1.4 \mathrm{a}$ & $1.1 \mathrm{a}$ & $2.7 \mathrm{a}$ & $11.4 \mathrm{a}$ \\
Valle & $8.1 \mathrm{~b}$ & $5.0 \mathrm{~b}$ & $2.3 \mathrm{~b}$ & $1.9 \mathrm{~b}$ & $12.8 \mathrm{~b}$ & $30.2 \mathrm{~b}$ \\
Montaña & $18.5 \mathrm{c}$ & $5.3 \mathrm{~b}$ & $3.1 \mathrm{~b}$ & $2.7 \mathrm{c}$ & $11.9 \mathrm{~b}$ & $41.6 \mathrm{c}$
\end{tabular}

Valores con la misma letra por columna son estadísticamente iguales, significancia de 0.05

El total de carbono fijado en el ambiente de montaña fue de $13 \mathrm{tC} / \mathrm{ha}$ anual, por lo tanto, estos sistemas superan a lo reportado por Chacón et al. (2007), quienes encontraron que los bosques secundarios de 15 años tienen una tasa de fijación de $3.1 \mathrm{t} / \mathrm{ha}$ de $\mathrm{C}$ por año. Asimismo, Ordóñez et al. (2001) reportan que los bosques de Pinus pseudostrobus tienen un potencial de captura de 0.8 t/ha de $\mathrm{C}$ por año, ya que en 250 años han logrado capturar $217 \mathrm{tC} / \mathrm{ha}$. Estos resultados indican que los sistemas de cultivo de maíz nativo superan las capacidades de fijación de carbono de los sistemas forestales, por lo que se constituyen en adecuados sumideros de carbono. Lo anterior concuerda con lo reportado por García et al. 2006, quienes mencionan que al ser el maíz una planta $\mathrm{C} 4$, posee altas tasas de actividad fotosintética, por lo que puede almacenar y mantener una gran cantidad de energía y biomasa, además de presentar muy poca o nula fotorrespiración (García et al. 2006, Hernández et al. 2008).

Jumilla et al. (2010), mencionan que el tiempo de permanencia del carbono retirado de la atmósfera es un aspecto fundamental del comportamiento de los reservorios agrícolas y de los sumideros en general. Reconocen que por muy pequeño que sea el periodo en el que el $\mathrm{CO}_{2}$ permanece almacenado en la vegetación agrícola, no invalida a ésta como reservorio, por lo que los cultivos de maíz nativo funcionan como una alternativa de fijación de carbono y, por ello, representan un servicio ambiental.

\section{CONCLUSIONES}

Los cultivos de maíz nativo producidos en montaña y en específico la variedad amarilla tienen una alta fijación de $\mathrm{CO}_{2}$ en sus estructuras $\left(34.37 \mathrm{tCO}_{2} \mathrm{eq} / \mathrm{ha}\right)$. Las características genéticas y las condiciones ambientales modifican el desarrollo, crecimiento, composición química y capacidad de fijación de carbono.

\section{AGRADECIMIENTOS}

Al Consejo Nacional de Ciencia y Tecnología (CONACyT) por la beca de estudios de posgrado otorgada a Marcos Solorio y por el financiamiento del trabajo experimental a través del proyecto "El maíz mesoamericano y sus escenarios en el desarro1lo local" No. de registro 130947. A la Universidad Autónoma de Sinaloa por el financiamiento a los trabajos experimentales realizados en la Facultad de Agronomía de dicha Universidad.

\section{REFEFENCIAS}

Aceves R. E., Turrent F. A., Cortés F. J. I. y Volke H. V. (2002). Comportamiento agronómico del híbrido H-137 y materiales criollos de maíz en el valle de puebla. Rev. Fitotec. Mex. 25, 339-347.

Amador R. A. L. y Boschini F. C. (2000). Fenología productiva y nutricional de maíz para la producción de forraje. Agron. Mesoam. 11, 171-177.

AOAC. (2006). Official Methods of Analysis of the Association of Official Analytical Chemists International. 13a Ed. The Association of Official Analytical Chemists. Arlington, EUA, 1038 pp.

Aragón C. F., Taba S., Hernández C. J. M., Figueroa C. J.D. y Serrano A. V (2006). Actualización de la información sobre los maíces criollos de Oaxaca. 
Instituto Nacional de Investigaciones Forestales, Agrícolas y Pecuarias. Sistema Nacional de Información sobre Biodiversidad- Comisión Nacional para el Conocimiento y Uso de la Biodiversidad. Ciudad de México, México, 133 pp.

Arellano V. J. L., Virgen V. J. y Ávila P. M. A. (2010). H-66 híbrido de maíz para los valles altos de los estados de México y Tlaxcala. Rev. Mex. Cienc. Agríc. $1,257-262$.

Bolaños G. Y., Bolaños G. M. A., Paz P. F., Gonalez W. J. y Barrales B. E. (2015). Estimación de carbono almacenado en dos bosques de referencia del Monte Tláloc en Texcoco, Estado de México. Memorias. Simposio Internacional del Carbono en México. Villahermosa, Tabasco. 20 al 22 de mayo. CD-ROM.

Casanova L. F., Petit A. J. y Solorio S. J. (2011).Los sistemas agroforestales como alternativa a la captura de carbono en el trópico mexicano. Rev. Chapingo Ser. Cienc. For. Am. 17, 5-118.

DOI: $10.5154 /$ r.rchscfa.2010.08.047.

Chacón P., Leblanc H.A. y Russo R.O. (2007). Fijación de carbono en un bosque secundario de la región tropical húmeda de Costa Rica. Tierra Trop. 3, 39-49.

CONAGUA (2015). Temperatura y lluvia.Resúmenes mensuales: consulta de tabla nacional por entidad federativa y mapas. Comisión Nacional del Agua. [en línea]. http://smn.conagua.gob.mx/index. php?option $=$ com_content\&view $=$ article\&id $=12 \&$ Ite mid=77 05/02/2015

Elizondo J. y Boschini C. (2002). Producción de forraje con maíz criollo y maíz híbrido. Agron. Mesoam. 13, 13-17.

Espinosa C. A., Tadeo R. M., Turrent F. A., Sierra M. M., Gómez M. N. y Zamudio G. B. (2013). Rendimiento de variedades precoces de maíz grano amarillo para valles altos de México. Agron. Mesoam. 24, 93-99.

Espinoza D. W., Krishnamurthy L., Vázquez A. A. y Torres R. A. (2012). Almacén de carbono en sistemas agroforestales con café. Rev. Chapingo Ser. Cienc. For. Am. 18, 57-70. DOI: 10.5154/r.rchscfa.2011.04.030.

Follett R.F. (2001). Soil management concepts and carbon sequestration in cropland soils. Soil Tillage Res. 61, 77-92. DOI: 10.1016/S0167-1987(01)00180-5.

García F., Roselló J. y Santamarina M. (2006). Introducción al funcionamiento de las plantas. Editorial de la Universidad Politécnica de Valencia. Valencia, España, $184 \mathrm{pp}$.

Jumilla F. V., Costa G. I., Castro C. T., García C. R., Romojaro C.C y Mesa del Castillo N. L. (2010). Balance de carbono en cultivos de agricultura intensiva. En: Iniciativas para una economía baja en carbono. (F. V. Jumilla, Ed.). Consejería de Agricultura y Agua. Murcia, España, pp. 214-276.
Méndez M. G., Solorza F. J., Velázquez V. M., Gómez M. N., Paredes L. O. y Bello P. L. A. (2005). Composición química y caracterización calorimétrica de híbridos y variedades de maíz cultivadas en México. Agrociencia $39,267-274$.

Ordóñez J. A., Bernardus H. J. J. y Masera O. (2001). Almacenamiento de carbono en un bosque de Pinus pseudostrobus en Nuevo San Juan, Michoacán. Madera Bosques 72, 27-47.

Paliwal R. L. (2001). Morfología del maíz tropical. En: El maíz en los trópicos: mejoramiento y producción. (R.L. Paliwal, G. Granados, H. R. Lafitte, A. D. Violic y J. P. Marathée, Eds). Organización de la Naciones Unidas para la Agricultura y la Alimentación. Departamento de Agricultura, Roma, Italia, 376 pp.

Pecina M. J. A., Mendoza C. M.C., López S. J. A., Castillo G. F., Mendoza R. M. y Ortiz C. J. (2011). Rendimiento de grano y sus componentes en maíces nativos de Tamaulipas evaluados en ambientes contrastantes. Rev. Fitotec. Mex. 34, 85-92.

Pérez C. F.J., Carballo C. A., Santacruz V. A., Hernández L. A. y Molina M. J. C. (2007). Calidad fisiológica en semillas de maíz con diferencias estructurales. Agric. Téc. Méx. 33, 53-61.

Robert M. (2002). Captura de carbono en los suelos para un mejor manejo de la tierra. Organización de las $\mathrm{Na}$ ciones Unidas para la Agricultura y la Alimentación. Roma, Italia, $70 \mathrm{pp}$.

SAS (1998). SAS/STATR User's guide. Release 6.03 edition. Statistical Analysis Software Institute Inc. Cary. Carolina del Norte, EUA, 364 pp.

Sierra D. C. (2010). Relación de la captura de carbono en Saccharum officinarum con otros factores ambientales para el cultivo de caña panelera. Tesis de Maestría. Instituto de Ciencias Ambientales. Universidad Nacional de Colombia. Bogotá, Colombia. 83 pp.

Tadeo R.M., Espinosa C. A., Zaragoza E. J., Turrent F. A., Sierra M. M. y Gómez M. N. (2012). Forraje y grano de híbridos de maíz amarillos para valles altos de México. Agron. Mesoam. 23, 281-288.

Vera G. A. M., Chávez S. J. L. y Carrillo R. J. C. (2012). Proteína, lisina y triptófano en poblaciones nativas de maíz mixteco. Rev. Fitotec. Mex. 35, 07-13.

Zepeda B. R., Carballo C. A., Muñoz O. A., Mejía C. J. A., Figueroa S. B., González C. F. V. y Hernández A. C. (2009). Proteína, triptófano y componentes estructurales del grano en híbridos de maíz (Zea mays L.) producidos bajo fertirrigación. Agrociencia 43, 143-152.

Zhang F., Mackenzie A. F. y Smith D. L. (1993). Corn yield and shifts among corn quality constituents following application of different nitrogen fertilizer sources at several times during corn development. J. Plant Nut. 16, 1317-1337. DOI: 10.1080/01904169309364615. 